\title{
The glitch activity of neutron stars (Corrigendum)
}

\author{
J. R. Fuentes ${ }^{1}$, C. M. Espinoza ${ }^{2}$, A. Reisenegger ${ }^{1}$, B. Shaw ${ }^{3}$, B. W. Stappers ${ }^{3}$, and A. G. Lyne ${ }^{3}$ \\ ${ }^{1}$ Instituto de Astrofísica, Pontificia Universidad Católica de Chile, Av. Vicuña Mackenna 4860, 7820436 Macul, Santiago, Chile \\ e-mail: jrfuentes@uc.cl \\ 2 Departamento de Física, Universidad de Santiago de Chile, Avenida Ecuador 3493, 9170124 Estación Central, Santiago, Chile \\ 3 Jodrell Bank Centre for Astrophysics, School of Physics and Astronomy, The University of Manchester, Manchester M13 9PL, UK
}

A\&A, 608, A131 (2017), https://doi.org/10.1051/0004-6361/201731519

Key words. stars: neutron - stars: magnetars - pulsars: general - stars: rotation - errata, addenda

The original article contains some errors, which are corrected below.

Introduction. Line of text number 10 , shows $\Delta \dot{v} / \dot{v} \sim 10^{-4}-10^{-3}$, but it must be " $\Delta \dot{v} / \dot{v} \sim 10^{-5}-10^{-2}$ ".

Figure 4. The $y$ axis is labeled by " $\log \dot{v}_{\mathrm{g}}\left(\mathrm{Hz} \mathrm{s}^{-1}\right)$ ", but it must be " $\dot{v}_{\mathrm{g}}\left(\mathrm{Hz} \mathrm{s}^{-1}\right)$ ".

Section 4.2. Page number 5, "the 7th bin $(\log |\dot{v}|=-10.75)$ " but it must be "7th bin $(\log |\dot{v}|=-13.75)$ ".

Equation (3) and its description is written as

$p_{i}=\min \left\{P\left(N_{\ell}^{\mathrm{obs}} \leq N_{\ell}^{\mathrm{exp}}\right), P\left(N_{\ell}^{\mathrm{obs}} \geq N_{\ell}^{\mathrm{exp}}\right)\right\}$, where $P\left(N_{\ell}^{\text {obs }} \leq N_{\ell}^{\text {exp }}\right)$ is the (Poisson) probability of observing a value $N_{\ell}^{\text {obs }}$ smaller or equal to the expected value $N_{\ell}^{\text {exp }}$, based on the fixed ratio $\dot{N}_{\ell} /|\dot{v}|=(4.2 \pm 0.5) \times 10^{2} \mathrm{~Hz}^{-1}$ calculated above (and analogously for $P\left[N_{\ell}^{\text {obs }} \geq N_{\ell}^{\exp }\right]$ ).

However, it must be

$p_{i}=\min \left\{P\left(x \leq N_{\ell}^{\mathrm{obs}}\right), P\left(N_{\ell}^{\mathrm{obs}} \geq x\right)\right\}$,

where $P\left(x \leq N_{\ell}^{\text {obs }}\right)$ is the (Poisson) probability of obtaining a value $x$ smaller or equal to the actual observed value $N_{\ell}^{\text {obs }}$, based on the fixed ratio $\dot{N}_{\ell} /|\dot{v}|=(4.2 \pm 0.5) \times 10^{2} \mathrm{~Hz}^{-1}$ calculated above, and the observation time of the pulsar (and analogously for $\left.P\left[N_{\ell}^{\mathrm{obs}} \geq x\right]\right)$.

Discussion. Line of text number 1 , shows " $\dot{v}<10^{-10.5} \mathrm{~Hz} \mathrm{~s}^{-1}$ " (3) but it must be " $|\dot{v}|<10^{-10.5} \mathrm{~Hz} \mathrm{~s}^{-1}$ ". 